\title{
Valoración de la docencia en un módulo de salud pública basado en competencias
}

\author{
M. Maldonado-Rojas
}

Introducción. Los cambios que se han producido en la educación superior invitan al docente a diseñar estrategias participativas de gestionar el proceso enseñanza-aprendizaje. Como una forma de evaluar los cambios que supone un nuevo modelo curricular, se sometió a valoración de la docencia un módulo de un plan curricular basado en competencias. El objetivo de esta evaluación era conocer si en el aula se impartía la docencia con base al modelo y cómo los alumnos valoraban las distintas estrategias usadas. Materiales y métodos. Se construyó un instrumento de recogida de la información. Los ítems consideraban el diseño específico del syllabus para el módulo en cuestión. El análisis de las respuestas se realizó a través de estadígrafos descriptivos y las respuestas abiertas fueron categorizadas; se recurrió a identificar fragmentos de frases reales escogidas según un criterio representativo del texto. Resultados. De forma general, los resultados demuestran la alta valoración que hacen de este módulo los estudiantes. Con relación a la preferencia de los alumnos por cada una de las metodologías empleadas, las que obtuvieron mayor porcentaje fueron trabajo colaborativo y talleres. El 46,3 (2006) y el 42,6\% (2007) de los estudiantes consideraron que todas las metodoligías usadas les ayudaron. Las distintas modalidades de evaluación son calificadas de manera similar, y la diferencia entre una y otra fue pequeña. Conclusiones. Es destacable la alta valoración del recurso 'plataforma WebCT', lo que demuestra que los estudiantes se motivan frente a la interacción que pueden desarrollar con el uso de las tecnologías de la información y la comunicación (TIC), así se sienten protagonistas y no receptores pasivos en el aula. Si se relaciona la metodología utilizada con las preferencias de los alumnos y con la importancia que le atribuyen a las capacidades adquiridas, se concluye que es muy relevante considerar varios aspectos en el momento de elegir la forma de cómo se desarrollarán las unidades de aprendizaje.

Palabras clave. Aprendizaje. Estrategias metodológicas. Evaluación de la docencia. Proceso de enseñanza.

\section{Assessment of a public health competences based topic}

Introduction. The changes suffered by the superior education invite teachers to design participative teaching strategies to carry out learning. As a way to evaluate changes for a new curriculum model, a curricular plan based on competencies was evaluated by the teachers. The objective of the evaluation was to know if teachers were really working with this approach and how students valued these different strategies. Materials and methods. According to the objective, a survey was designed. The items considered the specific syllabus for the evaluated module. Answer analysis was done by descriptive statistics and open answers were categorized identifying phrase segments chosen with a representative text criterion. Results. In general, results showed the high module evaluation done by the students. In relation to the students preferences for the performed methodologies, the best evaluated were: cooperative work and workshops. 46.3 (2006) and $42.6 \%$ (2007) considered them a very helpful methodology. Different ways of evaluating have no relevant differences. Conclusions. WebCt was highly marked by students what shows the importance of the interaction and TICS usage, marking them as main actors in the process and in the classroom. Methodology, skills acquired and students preferences are very important variables to consider when choosing how to develop the program units.

Key words. Evaluation teaching. Methodologic strategies. Process education-learning.

\section{Introducción}

Entre los cambios que se han producido en la educación superior después de iniciado el proceso de reforma, conocido como 'Tratado de Bolonia
Facultad de Ciencias de la Salud. Universidad de Talca. Talca, Chile.

\section{Correspondencia}

Dra. Mónica Maldonado Rojas. Facultad de Ciencias de la Salud. Universidad de Talca. Camino Lircay, s/n. Talca, Chile.

Fax (56-71) 200488

E-mail mamaldon@utalca.cl 
1999', una de las grandes líneas de acción que se debía desarrollar en el Espacio Europeo de Educación era la adopción de un sistema de titulaciones universitarias fácilmente comparable en toda Europa con la utilización de una valoración del crédito universitario igual para todos los países europeos (el llamado ECTS, European Credits Transfer System), junto con las adaptaciones curriculares que fomenten el aprendizaje continuado, la valoración del trabajo personal del alumno, las actividades no presenciales y la pérdida de protagonismo de la clase magistral [1]. Esta revolución en la enseñanza universitaria no sólo ocurre en Europa, sino también en Latinoamérica. Desde allí, se sugiere la aplicación en la enseñanza superior de métodos más centrados en los mecanismos de aprendizaje de los alumnos y se invita al docente a diseñar estrategias participativas de gestionar el proceso enseñanza-aprendizaje, junto con diseñar los currículos basados en la competencias necesarias para preparar a los estudiantes para el mundo laboral [2]. El hecho de formar profesionales realmente capacitados está hoy en día íntimamente ligado a la noción de aprendizaje como proceso abierto, flexible y permanente [3].

En el plano de las adaptaciones curriculares, a través de las competencias, se busca trascender del énfasis que la educación tradicional ha dado a la memorización de conocimientos descontextualizados, ya que éstas se basan en el análisis y la resolución de problemas con sentido para las personas, con flexibilidad, autonomía y creatividad [4]. Aportan una perspectiva que tiene impacto directo en la mejora de la calidad de la educación. Cada competencia es como un aprendizaje complejo que integra conocimientos, habilidades, aptitudes, valores y actitudes. Así entendida, se convierte en un dispositivo metodológico de construcción de los perfiles profesionales y disciplinarios, una discusión que debe ser incorporada en el diseño y rediseño de los programas, con la intención de mejorar la pertinencia de los modelos curriculares, en relación con los requerimientos cambiantes de la sociedad en general y del mundo del trabajo en particular [5].

Los profesionales que imparten docencia en las universidades se ven obligados a pasar de ser especialistas en ciencia a ser profesionales del aprendizaje de las materias de sus disciplinas [6], que no es otra cosa que añadir competencias pedagógicas a las competencias científicas para estimular el aprendizaje en los alumnos.
Uno de los retos que el profesor universitario debe afrontar es realizar de forma adecuada la mediación didáctica necesaria para facilitar la conexión de nuevos contenidos con el conocimiento previo con que parte el alumno, para construir sobre éstos el nuevo saber, con la precaución de no cargar de contenidos y reflexionando más sobre la calidad del aprendizaje que ha conseguido el alumno. La reducción de los temarios a núcleos fundamentales es una recomendación que emana del modelo propuesto por la convergencia europea junto con la racionalización de las horas de estudio y trabajo que se exigen al alumnado. Además, se propone un formato más funcional que promueva la intervención del alumno en la búsqueda, selección y comprensión de la información, lo que en la práctica les permitirá construir su aprendizaje, elaborar conclusiones personales y entender que los procesos de aprendizaje son construcciones sociales, compartidas, y que la responsabilidad de lo que ocurre en el aula no es sólo tarea exclusiva del profesor [2].

Otra de las modificaciones que se derivan del nuevo modelo es la diversificación de las metodologías, para atender a mayor número de estilos de aprendizaje, dada la diversidad y pluralidad del alumnado, de tal forma que sea posible alcanzar una competencia determinada siguiendo caminos formativos diferentes. La metodología que hay que emplear debe ser variada, flexible y contextualizada.

Finalmente, la evaluación también se ve afectada en este cambio de rol del docente universitario, que pasa de una valoración sólo de resultados y muchas veces centrada en lo cognitivo a una evaluación continua, en la que se valora el proceso y de esta forma ayuda a mejorar el aprendizaje. La evaluación debe ser integral, de tal modo que abarque competencias cognitivas y permita además evaluar habilidades, destrezas $\mathrm{y}$ actitudes.

Además, es necesario no centrar la evaluación sólo en los alumnos, y evaluar también la calidad de la labor del profesor, la planificación, el contexto y los recursos empleados, teniendo en cuenta que el proceso de aprendizaje no es responsabilidad sólo del estudiante, sino que deben considerarse los diferentes agentes del proceso formativo.

En el contexto de la educación chilena, en 1997, el gobierno de Chile definió una nueva política 
de desarrollo de la educación superior, creando el Programa de Mejoramiento de la Calidad y la Equidad de la Educación Superior (MECESUP), que apunta a complementar el proceso de reforma de la educación iniciado en la última década. Las políticas públicas que de ahí emanan dan orientaciones claras, focalizando su apoyo a la renovación curricular con modelos basados en competencias.

En los últimos años, la Universidad de Talca ha entrado en el proceso de renovación curricular como una decisión corporativa, que ha sido pionera en el país. El proceso llevado a cabo desde el año 2002, con apoyo del programa MECESUP, comenzó a ponerse en marcha en 2006. Este proceso ha permitido involucrar a los docentes universitarios, aunque también ha desatado resistencias al cambio curricular, ya que implica necesariamente consecuencias sobre las actividades que los docentes desarrollan en las aulas [7].

Controlar el proceso se torna entonces fundamental para que esta propuesta de cambio y los hechos, que dan forma al currículo y que ocurren en las aulas, no queden en la práctica como una continuación de lo mismo con la apariencia de cambio.

Como una forma de iniciar el proceso de evaluación de los cambios que supone el nuevo modelo curricular impartido en la Universidad de Talca, uno de los módulos del nuevo plan curricular de la carrera de Tecnología Médica se sometió a valoración de la docencia. En Chile, esta carrera se imparte en las universidades y tiene una duración de cinco años. El perfil profesional definido por la Comisión Nacional de Acreditación de Pregrado (CNAP), en términos generales, señala que: 'El tecnólogo médico es un profesional cuya formación está sustentada en principios éticos y humanistas. Posee sólidos conocimientos y comprensión de los fundamentos biológicos, biofísicos y bioquímicos que le permiten desarrollar competencias y habilidades para actuar en los ámbitos de prevención, fomento y recuperación de la salud, mediante exámenes y procedimientos, destinados al diagnóstico y tratamiento de patologías que afectan al hombre y su entorno' [8]. El Plan de Estudios de la Universidad de Talca lo habilita para que se desempeñe principalmente en instituciones de salud, tanto públicas como privadas, donde podrá cumplir funciones en el área asistencial, administración, educación e investigación científica. Además, su rigurosa formación en el trabajo de laboratorio lo habilita para tra- bajar en centros de biotecnología, farmacéutica o industrias de alimentos, entre otras.

Los módulos que configuran el itinerario curricular de la formación profesional están diseñados de manera que la secuencia de éstos permite que se desarrollen las capacidades que finalmente llevarán al logro de las competencias. Estos módulos están clasificados en componentes de la formación fundamental, básica y disciplinaria. El módulo Salud Pública es el primer módulo correspondiente a la formación disciplinaria que enfrenta el alumno, y es éste el que se sometió a valoración de la docencia por parte de los alumnos. El objetivo de esta evaluación era conocer si en el aula se impartía la docencia con base al nuevo modelo y cómo los alumnos valoraban las distintas estrategias usadas.

\section{Materiales y métodos}

Para los efectos de este estudio se construyó un instrumento de recogida de información. Los ítems consideraban el diseño específico del $s y$ llabus para el módulo en cuestión. Así es cómo se consultaba sobre aspectos como organización, número de horas, metodologías empleadas, uso del WebCT, modalidades de evaluación, unidades de aprendizajes abordadas, cumplimiento de la actividad docente, entre otras. El diseño de este estudio fue de corte transversal, su grado de control era mínimo. El grupo encuestado estuvo formado por los estudiantes que cursaron el módulo Salud Pública correspondiente al primer nivel de la carrera de Tecnología Médica en la Universidad de Talca. Los alumnos encuestados fueron 54 y 55 en el año 2006 y 2007, respectivamente. El cuestionario constaba de preguntas con escala codificada y preguntas abiertas, en el que los alumnos dieron su opinión en función de las variables consultadas. Se pidió que calificaran cada indicador en una escala de 1 a 7 . Se dejó un espacio para las observaciones, que se incorporaron al final del instrumento. Para ambos cursos, los cuestionarios se repartieron en la última sesión presencial, con todas las actividades evaluativas realizadas, con la mayoría de sus calificaciones conocidas, y sólo quedó pendiente la calificación final obtenida.

El análisis de las respuestas se realizó a través de los estadígrafos descriptivos: media, desviación 
estándar o porcentaje. Las respuestas abiertas se establecieron por categorías y se escogieron fragmentos de frases reales según un criterio como representador del texto.

\section{Resultados}

En el año 2006 el total de alumnos que cursó el módulo Salud Pública fue de 55 y se encuestó a 54. En el año 2007 el total de alumnos que cursó el módulo fue de 70 y se encuestó a 55. Los resultados obtenidos para los ítems fueron: comentarios generales sobre el módulo, comentarios sobre el desarrollo del módulo y cumplimiento de la actividad docente, con cada una de sus variables promediadas e incluida su variabilidad (Tabla I).

La tabla II muestra los resultados obtenidos de acuerdo con el porcentaje de alumnos que opinó sobre cada una de las unidades específicas del módulo en cuanto a: unidad más interesante, unidad que podría ser mejorada y unidad en la que se siente más inseguro.

En la tabla III se muestran los resultados obtenidos en porcentajes sobre la preferencia de los alumnos en metodologías empleadas. La tabla IV muestra los resultados sobre la calificación otorgada por los alumnos a la docencia del módulo Salud Pública y se acompaña con el promedio y diversidad de las calificaciones obtenidas por los alumnos en dicho módulo.

\section{Observaciones realizadas por los alumnos}

Por último, se examinan las respuestas de los encuestados en el espacio 'Observaciones'. Éstas fueron categorizadas, recurriendo a identificar fragmentos de frases reales escogidas según un criterio como representador del texto, y en función de su naturaleza se agruparon cada año en tres categorías.

En el grupo de alumnos del año 2006 se identificaron las siguientes categorías: un primer grupo, con comentarios que tienen relación con la programación del módulo; una segunda categoría son los comentarios realizados a modo de sugerencias, y una tercera con los comentarios dirigidos a la labor docente.

En el año 2007 se identificaron, como primer grupo, comentarios que tenían relación con los recursos utilizados, un segundo grupo incluía los comentarios atribuibles a las dificultades y debilidades encontradas, y un tercer grupo con los comentarios hechos a la labor docente. En la tabla $\mathrm{V}$ se muestra una síntesis de los comentarios recibidos en cada categoría para cada año.

\section{Discusión}

El documento que expresa la planificación del módulo es el syllabus, en el que se indican y organizan los propósitos de aprendizaje, donde se fija el tiempo de trabajo del alumno, junto con la selección de las metodologías, tanto de la formación como de la evaluación de las competencias; se considera como instrumento guía no sólo para el profesor, sino también para el estudiante, ya que explicita el rol que tendrá en su proceso. Se diseñó para hacer este estudio, una pauta de valoración de la docencia basándose en el syllabus entregado para el módulo Salud Pública de la carrera de Tecnología Médica de la Universidad de Talca (todos los syllabus son sancionados previamente por el consejo de escuela). Se manifiesta que se parte con el control de la puesta en marcha de un currículo rediseñado, donde el módulo evaluado es el primero que conoce el alumno de su formación disciplinaria al ingresar en la universidad.

Existen varias limitaciones del estudio, como son: la construcción del instrumento que se hizo basándose solamente en el syllabus del módulo en evaluación, sin que haya existido una validación; la autoaplicabilidad del instrumento, lo que no asegura la total comprensión de las preguntas, dado que no existió una prueba piloto que evidenciara ésta por parte de los alumnos; otra limitación es que la encuesta fue anónima, por lo que no se puede realizar un análisis de la valoración que hace cada alumno de acuerdo a la calificación obtenida, y finalmente no se midió la capacidad de 'valorar' de los alumnos, aunque se destaca que no se encontró ambivalencias en sus respuestas.

Si consideramos las limitaciones del diseño del estudio, se hace una descripción de los datos interpretando la media como medida de tendencia central y la desviación estándar como variabilidad, en conjunto. Con relación a los resultados obtenidos y siendo el objetivo de esta evaluación conocer si en el aula se impartía la docencia con 
Tabla I. Calificación de las variables consultadas del módulo Salud pública, como promedio para cada una, años 2006-2007.

\begin{tabular}{|c|c|c|c|c|}
\hline & \multicolumn{4}{|c|}{ Calificación } \\
\hline & \multicolumn{2}{|c|}{$2006(n=54)$} & \multicolumn{2}{|c|}{$2007(n=55)$} \\
\hline & Media & $\mathrm{DE}$ & Media & $\mathrm{DE}$ \\
\hline \multicolumn{5}{|l|}{ I. Comentarios generales sobre el módulo } \\
\hline Cumplimiento en el desarrollo de las competencias comprometidas en el syllabus & 6,6 & 0,62 & 6,7 & 0,53 \\
\hline Cumplimiento de sus expectativas personales & 6,1 & 0,90 & 6,1 & 0,84 \\
\hline Claridad en el propósito de los aprendizajes del módulo a & - & - & 6,6 & 0,55 \\
\hline \multicolumn{5}{|l|}{ II. Comentarios sobre el desarrollo del módulo } \\
\hline Las unidades de aprendizaje son relevantes para iniciar su formación profesional & 6,4 & 0,87 & 6,7 & 0,58 \\
\hline La metodología usada permite desarrollar nuevas competencias & 6,1 & 1,03 & 6,4 & 0,63 \\
\hline $\begin{array}{l}\text { La organización práctica del módulo en cuanto a la logística } \\
\text { (material entregado, medios, plataforma, etc.) }\end{array}$ & 6,7 & 0,48 & 6,7 & 0,49 \\
\hline $\begin{array}{l}\text { La incorporación del curso a la plataforma WebCT } \\
\text { le ha ayudado en la organización de sus estudios }\end{array}$ & 6,8 & 0,51 & 6,7 & 0,64 \\
\hline $\begin{array}{l}\text { La forma de integrar las herramientas: contenidos, metodologías, formas de } \\
\text { evaluación, permiten el desarrollo de competencias declaradas en el syllabus }\end{array}$ & 6,3 & 0,75 & 6,3 & 0,67 \\
\hline Conocer las pautas de evaluación con anterioridad le sirve para guiar su aprendizaje ${ }^{a}$ & - & - & 6,5 & 1,00 \\
\hline Las evaluaciones del módulo permiten retroalimentación y ayudan en su aprendizaje & 6,3 & 0,72 & 6,4 & 0,66 \\
\hline $\begin{array}{l}\text { Combinar distintas formas de evaluación permite demostrar distintas capacidades } \\
\text { y no sólo conocimientos }{ }^{\text {a }}\end{array}$ & - & - & 6,6 & 0,61 \\
\hline
\end{tabular}

Califique de forma independiente cada una de las modalidades de evaluación utilizadas:

$\begin{array}{lllll}\text { Talleres } & 6,3 & 0,72 & 6,5 & 0,68 \\ \text { Informes } & 6,2 & 0,88 & 6,2 & 0,74 \\ \text { Exposición oral } & 6,2 & 0,79 & 6,5 & 0,78 \\ \text { Trabajos grupales } & 6,3 & 0,85 & 6,5 & 0,75 \\ \text { Pruebas escritas } & 6,9 & 1,02 & 6,4 & 0,67 \\ \text { Autoevaluación y evaluación de pares } & 6,5 & 0,63 & 6,2 & 0,99\end{array}$

\section{Cumplimiento de la actividad docente}

\begin{tabular}{|c|c|c|c|c|}
\hline La disposición de los docentes para atender a los alumnos & 6,9 & 0,29 & 6,8 & 0,36 \\
\hline $\begin{array}{l}\text { El cumplimiento de sus labores docentes } \\
\text { (programas, puntualidad, entrega de evaluaciones, etc.) }\end{array}$ & 6,7 & 0,47 & 6,7 & 0,34 \\
\hline $\begin{array}{l}\text { La capacidad de contribuir, en alguna medida a la formación del estudiante } \\
\text { (pensamiento, valores, conductas, hábitos y disciplina) }\end{array}$ & 6,6 & 0,68 & 6,8 & 0,62 \\
\hline
\end{tabular}

DE: desviación estándar. ${ }^{a}$ Variables incorporadas en la segunda versión del documento. 
Tabla II. Opinión de los alumnos sobre las unidades específicas del módulo, años 2006-2007.

\begin{tabular}{|c|c|c|c|}
\hline & & $2006(n=54)$ & $2007(n=55)$ \\
\hline \multirow{6}{*}{$\begin{array}{l}\text { Unidad más } \\
\text { interesante }\end{array}$} & U1: La salud como fenómeno social & $14,3 \%$ & $11,1 \%$ \\
\hline & U2: Sistema sanitario y seguridad social chilena & $20,0 \%$ & $7,4 \%$ \\
\hline & U3: Epidemiología & $28,6 \%$ & $33,3 \%$ \\
\hline & U4:Tecnología médica como ciencia de la salud & $34,3 \%$ & $44,7 \%$ \\
\hline & Todas & $2,8 \%$ & $3,7 \%$ \\
\hline & No responde & $0,0 \%$ & $0,0 \%$ \\
\hline \multirow{5}{*}{$\begin{array}{l}\text { Unidad } \\
\text { que podría } \\
\text { mejorarse }\end{array}$} & U1: La salud como fenómeno social & $5,7 \%$ & $16,7 \%$ \\
\hline & U2: Sistema sanitario y seguridad social chilena & $11,4 \%$ & $22,2 \%$ \\
\hline & U3: Epidemiología & $31,4 \%$ & $18,5 \%$ \\
\hline & U4:Tecnología médica como ciencia de la salud & $5,7 \%$ & $7,4 \%$ \\
\hline & Ninguna & $45,7 \%$ & $35,2 \%$ \\
\hline \multirow{6}{*}{$\begin{array}{l}\text { Unidad que le } \\
\text { ha quedado } \\
\text { confusa, } \\
\text { con menos } \\
\text { confianza en } \\
\text { lo aprendido }\end{array}$} & U1: La salud como fenómeno social & $0,0 \%$ & $11,1 \%$ \\
\hline & U2: Sistema sanitario y seguridad social chilena & $4,8 \%$ & $20,4 \%$ \\
\hline & U3: Epidemiología & $19,0 \%$ & $13,0 \%$ \\
\hline & U4:Tecnología médica como ciencia de la salud & $0,0 \%$ & $0,0 \%$ \\
\hline & Ninguna & $76,2 \%$ & $50,0 \%$ \\
\hline & No responde & $0,0 \%$ & $5,6 \%$ \\
\hline
\end{tabular}

base al nuevo modelo y cómo los alumnos valoraban las distintas estrategias usadas, se puede concluir que, en primer lugar, no existen diferencias importantes en la valoración que hacen los alumnos que evaluaron el módulo, en los dos años en que fue aplicado el instrumento, por lo que no se hará distinción entre uno u otro año. De forma general, los resultados demuestran la alta valoración que hacen de este módulo, puesto que ningún indicador está por debajo de la calificación 6,0 y no existen desviaciones mayores a 1,03 unidades de la escala.

A modo de comentario de algunos aspectos de la evaluación de la docencia: para el indicador 'cumplimiento en el desarrollo de las competencias comprometidas en el syllabus', califican con 6,6 y 6,7 para el año 2006 y 2007, respectivamente, lo que es indicativo que lo declarado como competencias que hay que lograr son percibidas como adquiridas por parte de los alumnos. En cuanto al 'cumplimiento de sus expectativas personales', en ambos años es el indicador que presenta una de las calificaciones más bajas $(6,1)$, lo que podría atribuirse a que los estudiantes comienzan su formación profesional con gran entusiasmo, expectantes a lo que será esta nueva etapa y muchos tal vez con desconocimiento de la carrera a la que han optado. 
Tabla III. Opinión de los alumnos sobre las metodologías empleadas, años 2006-2007.

\begin{tabular}{|c|c|c|c|}
\hline & & $2006(n=54)$ & $2007(n=55)$ \\
\hline \multirow{8}{*}{$\begin{array}{l}\text { Metodología empleada, } \\
\text { que considera que le ayudó } \\
\text { más en su proceso de aprendizaje }\end{array}$} & Informes & $18,6 \%$ & $11,1 \%$ \\
\hline & Talleres & $20,4 \%$ & $7,4 \%$ \\
\hline & Trabajo colaborativo & $38,7 \%$ & $33,3 \%$ \\
\hline & Exposiciones orales & $6,1 \%$ & $44,7 \%$ \\
\hline & Lecturas $^{a}$ & - & $0,0 \%$ \\
\hline & Clases & $6,9 \%$ & $7,4 \%$ \\
\hline & Todas & $7,4 \%$ & $5,6 \%$ \\
\hline & No responde & $1,9 \%$ & $9,2 \%$ \\
\hline \multirow{8}{*}{$\begin{array}{l}\text { Metodología empleada, } \\
\text { que considera que le ayudó } \\
\text { menos en su proceso de aprendizaje }\end{array}$} & Informes & $3,7 \%$ & $9,3 \%$ \\
\hline & Talleres & $11,1 \%$ & $3,7 \%$ \\
\hline & Trabajo colaborativo & $11,1 \%$ & $7,4 \%$ \\
\hline & Exposiciones orales & $13,0 \%$ & $11,1 \%$ \\
\hline & Lecturas $^{a}$ & - & $3,7 \%$ \\
\hline & Clases & $7,4 \%$ & $14,8 \%$ \\
\hline & Todas & $46,3 \%$ & $42,6 \%$ \\
\hline & No responde & $7,4 \%$ & $7,4 \%$ \\
\hline
\end{tabular}

Con relación al desarrollo del módulo, recibieron la valoración más alta los siguientes aspectos: 'la organización práctica del módulo en cuanto a la logística (material entregado, medios, etc.) y la incorporación del curso a la plataforma WebCT como ayuda en la organización de sus estudios', lo que puede ser interpretado como que los estudiantes se motivan frente a la interacción que pueden desarrollar con el uso de las TIC, sintiéndose protagonistas y no meros receptores pasivos en el aula.

Los indicadores que tienen relación con la forma de evaluar las competencias adquiridas, de los cuales dos fueron incorporados en la segunda versión del instrumento, también son bien valorados por los estudiantes, con una calificación de 6,5 para 'conocer las pautas de evaluación con anterioridad le sirve para guiar su aprendizaje, de 6,4 para 'las evaluaciones del módulo permiten retroalimentación y ayudan en su aprendizaje' y de 6,6 para 'combinar distintas formas de evaluación permite demostrar distintas capacidades y no sólo conocimientos', en el grupo encuestado el 2007. Esto se corrobora con los comentarios realizados por los alumnos en el apartado Observaciones, en el que destacan como positivo el conocimiento de las pautas y la variedad de metodologías utilizadas para la evaluación. 
Tabla IV. Calificación otorgada por los alumnos a la docencia del módulo y calificación obtenida por los alumnos en el módulo Salud Pública, años 2006-2007.

\begin{tabular}{|c|c|c|c|c|c|c|}
\hline & \multicolumn{3}{|c|}{$2006(n=54)$} & \multicolumn{3}{|c|}{$2007(n=55)$} \\
\hline & \multicolumn{2}{|c|}{ Media } & $\mathrm{DE}$ & \multicolumn{2}{|c|}{ Media } & DE \\
\hline Calificación general para el módulo & \multicolumn{2}{|c|}{6,2} & 0,62 & \multicolumn{2}{|c|}{6,3} & 0,50 \\
\hline \multirow{2}{*}{$\begin{array}{l}\text { Considera que con base al conocimiento adquirido } \\
\text { en este módulo se producirán cambios en la forma } \\
\text { de enfrentar su formación universitaria y la manera } \\
\text { de relacionarse con sus profesores y compañeros }\end{array}$} & Sí & No & NR & Sí & No & NR \\
\hline & $57,4 \%$ & $5,6 \%$ & $37 \%$ & $77,8 \%$ & $9,3 \%$ & $14,8 \%$ \\
\hline \multirow{2}{*}{$\begin{array}{l}\text { Calificación promedio obtenida } \\
\text { por los alumnos en el módulo }\end{array}$} & \multicolumn{2}{|c|}{ Media } & Rango & \multicolumn{2}{|c|}{ Media } & Rango \\
\hline & \multicolumn{2}{|c|}{5,3} & $4,2-6,1$ & \multicolumn{2}{|c|}{5,2} & $4,4-6,0$ \\
\hline
\end{tabular}

DE: desviación estándar; NR: no responde.

Las distintas modalidades de evaluación son calificadas de manera similar y la diferencia entre una y otra es pequeña.

En relación con la opinión de los alumnos sobre las unidades específicas del módulo y sobre metodologías empleadas en cada una de ellas, se concluye que la unidad que concita el mayor interés en ellos, dado que fue considerada como la más interesante por el 34,3 y el $44,7 \%$ de los alumnos en el 2006 y 2007, respectivamente, es la denominada 'Tecnología médica como ciencia de la salud'. En ésta el alumno conoce las características específicas y el campo de la tecnología médica como disciplina dentro de las ciencias de la salud, y el rol que le corresponderá desempeñar como profesional del área; además puede identificar ámbitos de desempeño, orientarse en la futura formación profesional, conocer el código deontológico de los profesionales de la salud y comprender la importancia de actuar ética y moralmente con el mayor respeto por la vida humana y la importancia de la confidencialidad en el ámbito de su profesión. Esto es indicativo de que los alumnos valoran conocer la profesión para la que empiezan a formarse, el contacto con el ámbito laboral y demuestra además que no siempre tienen conocimiento cabal de la disciplina a la que han optado para formarse profesionalmente. Esto se reafirma con los comentarios realizados a modo de observaciones, donde claramente agradecen la visión que se les da de la carrera. Por otra parte, para el grupo del año 2007, la unidad que resulta menos interesante, también declarada como la que debiera mejorarse, y considerada, además, como aquella en la que los conocimientos están más confusos y por ende sienten mayor inseguridad, es la denominada 'Sistema sanitario y seguridad social chilena. Cabe señalar que una de las metodologías usadas en esta unidad fue la exposición oral ante pares, y en este mismo grupo es considerada como aquella metodología que menos aportó al aprendizaje.

Siguiendo con las metodologías empleadas, las que obtuvieron mayor porcentaje fueron: trabajo colaborativo $(38,7 \%$ y $40,7 \%)$ y talleres $(20,4 \%$ y $29,6 \%$ ) en los grupos 2006 y 2007, respectivamente. Un alto porcentaje de estudiantes también consideró que todas les ayudaron (46,3 y 42,6\%, en 2006 y 2007, respectivamente). El trabajo colaborativo fue la metodología utilizada para la unidad 2 y 4 en el año 2006 y la unidad 4 el año 2007. El método empleado en las unidades 1, 3 y 4 en ambos años fueron los talleres.

Al hacer una relación entre la metodología utilizada en cada unidad de aprendizaje y los resultados obtenidos en los indicadores relacionados con las preferencias de los alumnos y con la importancia que le atribuyen a las capacidades adquiridas en cada una de ellas, y si se toman en cuenta además las observaciones realizadas, se concluye que es muy relevante considerar varios 
Tabla V. Comentarios según las categorías, años 2006 y 2007.

\begin{tabular}{|c|c|c|}
\hline & Categorías & Comentarios \\
\hline \multirow{3}{*}{$\begin{array}{l}\text { Año } \\
2006\end{array}$} & $\begin{array}{l}\text { Programación } \\
\text { del módulo }\end{array}$ & $\begin{array}{l}\text { El trabajo colaborativo les obliga a ir sobre el terreno en busca de información } \\
\text { y les permite conocer mejor a sus compañeros, además de enseñarles a organizarse } \\
\text { Consideran positivo la variedad de metodologías evaluativas y de ayuda para el aprendizaje } \\
\text { El uso del recurso de la plataforma WebCT se considera de mucha ayuda } \\
\text { El módulo no sólo les entrega herramientas para la formación, sino también sociales }\end{array}$ \\
\hline & Sugerencias & $\begin{array}{l}\text { Realizar más talleres, pues permite el acceso directo al profesor para aclarar las dudas } \\
\text { en el momento } \\
\text { Salidas sobre el terreno para conocer los distintos ámbitos de la profesión } \\
\text { Que los trabajos de grupo sean mejor controlados para que todos aporten por igual }\end{array}$ \\
\hline & Labor docente & $\begin{array}{l}\text { Las docentes ayudan a afrontar mejor el primer semestre de formación en la universidad, } \\
\text { dan una visión clara de lo qué es la profesión y muestran cómo incorporar lo que se } \\
\text { aprende; agradecen la buena disposición de las docentes }\end{array}$ \\
\hline \multirow{3}{*}{$\begin{array}{l}\text { Año } \\
2007\end{array}$} & $\begin{array}{l}\text { Recursos } \\
\text { utilizados }\end{array}$ & $\begin{array}{l}\text { Apoyan la iniciativa de usar la plataforma WebCT } \\
\text { Valoran conocer las pautas de evaluación con anterioridad } \\
\text { Valoran la variedad de metodologías empleadas en el proceso y en la evaluación, } \\
\text { lo que les permite demostrar y desarrollar capacidades distintas }\end{array}$ \\
\hline & $\begin{array}{l}\text { Dificultades } \\
\text { y debilidades } \\
\text { encontradas }\end{array}$ & $\begin{array}{l}\text { Las lecturas se hacen difíciles de entender, dado que al iniciar la formación profesional } \\
\text { hay muchos términos que desconocen } \\
\text { La metodología utilizada en la unidad } 2 \text { (trabajo grupal y exponer ante pares) } \\
\text { les deja 'cojos' en algunos temas, que no son bien abordados por los grupos } \\
\text { Solicitan más talleres de ejercicios en epidemiología y más salidas sobre el terreno } \\
\text { para conocer los ámbitos laborales de la profesión }\end{array}$ \\
\hline & Labor docente & $\begin{array}{l}\text { Agradecimientos por la forma en que fue realizado el módulo, por la relación interpersonal } \\
\text { desarrollada con las profesoras, por la orientación dada, por prepararlos para enfrentar } \\
\text { la formación profesional no como un alumno más, si no como un futuro profesional, } \\
\text { conociendo claramente el papel que deben desempeñar en el equipo de salud }\end{array}$ \\
\hline
\end{tabular}

aspectos en el momento de elegir la forma de desarrollar las unidades de aprendizaje, en cuanto a metodología y evaluación, de acuerdo con las capacidades que se quieren lograr, el número de alumnos, las características del grupo y las condiciones de entrada de los estudiantes.

Para la valoración de la labor docente, los tres indicadores utilizados presentan calificación entre 6,6 y 6,9 en ambos años, y se reafirma esta valoración con los comentarios de los alumnos. Finalmente, la calificación global que le dan a este módulo es de 6,2 y 6,3 para los años 2006 y 2007 , respectivamente, y un 57,4\% de alumnos en 2006 y un $77,8 \%$ de alumnos en 2007 consideran que haber realizado este módulo les ayudaría en la forma de enfrentar su formación universitaria y en la manera de relacionarse con sus profesores y compañeros.
Las calificaciones finales obtenidas por los alumnos en este módulo fueron como promedio del curso del 5,3, con un intervalo de 4,4-6,0, y una nota promedio del 5,2 , con un intervalo de 4,2-6,1 para los años 2006 y 2007, respectivamente (la Universidad de Talca utiliza escala numérica del 1,0 al 7,0, y la nota mínima de aprobado es el 4,0$)$. No se hace un estudio de correlación entre ambas variables, dado que la encuesta realizada a los alumnos fue de carácter anónimo, por lo que sólo se conoce la identidad del alumno en la nota de aprobado, pero se desconoce cuál fue la calificación que éste mismo dio a la docencia. Sin embargo, se puede observar que los alumnos valoraron positivamente la estrategia docente independiente de sus calificaciones.

Para concluir esta primera instancia de evaluación, desde la perspectiva de los alumnos, del 
proceso de instauración del nuevo modelo curricular en el módulo Salud Pública de la carrera de Tecnología Médica de la Universidad de Talca, se demuestra que se están haciendo esfuerzos para concretar los cambios planteados por el nuevo modelo implementado en la institución para no quedar sólo en la declaración de nuevas prácticas docentes y por otro lado que los alumnos están incorporando esta nueva estrategia formativa.

Conocer la opinión de los alumnos servirá de base para mejorar la labor docente en cuanto a la gestión del proceso enseñanza-aprendizaje, como lo es la entrega de contenidos, la utilización de recursos, el uso de las estrategias metodológicas y evaluativas y las relaciones profesor-alumno.

\section{Bibliografía}

1. Ceballos D, Cantarero D, Pascual M. El Tratado de Bolonia y la Enseñanza Superior: una experiencia comparada de introducción de las TIC en Ciencias Económicas. Barcelona: EDUTEC; 2004. URL: http://edutec2004.lmi. ub.es/pdf/134.pdf. [09.07.2007].
2. Mérida R. Nueva percepción de la identidad profesional del docente universitario ante la convergencia europea. Revista electrónica de Investigación Educativa 2006 URL: http://redie.uabc.mx/vol8no1/contenido-merida. html. [09.07.2007].

3. Breedlove $\mathrm{C}$, Hedrick H. Reenvisioning medical education for the new millennium. JAMA 1999; 282: 84-92.

4. González J, Wagenaar R, eds. Tuning educational structures in Europe. Informe final. 2003. Universidad de Deusto-Universidad de Groningen. La calidad y los enfoques de enseñanza y aprendizaje, evaluación y rendimiento. 2003; 4: 59-62.

5. INEM. Metodología para la Ordenación de la Formación Profesional Ocupacional. Madrid; 1995.

6. Zabalza MA. Competencias docentes del profesorado universitario. Calidad y desarrollo profesional. 1. ${ }^{\mathrm{a}}$ ed. Madrid: Narcea; 2003.

7. Corvalán $\mathrm{O}$, Hawes G. Aplicación del enfoque de competencias en la construcción curricular de la Universidad de Talca. Talca: Universidad de Talca. Documento de Trabajo IIDE-Proyecto MECESUP TAL101; 2006.

8. Leiva ME, Maldonado RM, Vásquez RM, Vidal FS. Proceso de rediseño curricular basado en competencias de la carrera de Tecnología Médica. Talca: Universidad de Talca; 2006. 